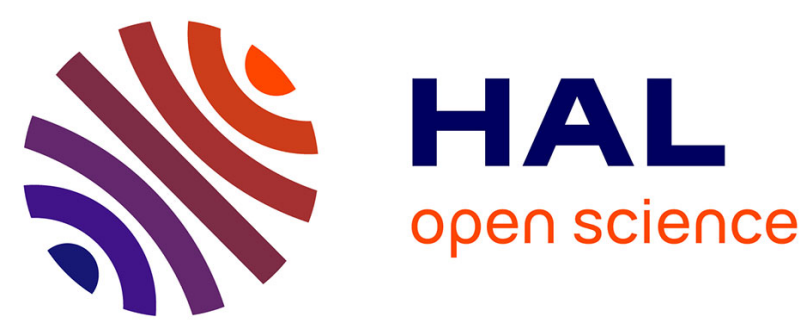

\title{
Le sanctuaire de Gournay-sur-Aronde (Oise) : structures et rites, les animaux du sacrifice \\ Jean-Louis Brunaux, Patrice Méniel
}

\section{To cite this version:}

Jean-Louis Brunaux, Patrice Méniel. Le sanctuaire de Gournay-sur-Aronde (Oise) : structures et rites, les animaux du sacrifice. Revue archéologique de Picardie, 1983, Les Celtes dans le nord du bassin parisien. Actes du Ve colloque de l'Association française pour l'étude de l'âge du Fer, 1, pp.165-173. 10.3406/pica.1983.2993. hal-02536436

\section{HAL Id: hal-02536436 \\ https://hal.science/hal-02536436}

Submitted on 8 Apr 2020

HAL is a multi-disciplinary open access archive for the deposit and dissemination of scientific research documents, whether they are published or not. The documents may come from teaching and research institutions in France or abroad, or from public or private research centers.
L'archive ouverte pluridisciplinaire HAL, est destinée au dépôt et à la diffusion de documents scientifiques de niveau recherche, publiés ou non, émanant des établissements d'enseignement et de recherche français ou étrangers, des laboratoires publics ou privés.

\section{(ㅇ)(1) $\$$}

Distributed under a Creative Commons Attribution - NonCommercial - NoDerivatives| 4.0 


\title{
LE SANCTUAIRE DE GOURNAY-SUR-ARONDE (Oise) : STRUCTURES ET RITES LES ANIMAUX DU SACRIFICE
}

\author{
par Jean-Louis BRUNAUX * et Patrice MENIEL **
}

On ne saurait faire, en quelques lignes, le point des connaissances sur le sanctuaire de Gournay. Une publication exhaustive sur les structures et leur interprétation aura cette mission ; elle paraîtra au cours de l'année 1983 (Brunaux, 1983). Pour I'heure, nous rappellerons, à grands traits, les aspects de la découverte. Nous nous attacherons aussi plus particulièrement à en dégager l'apport pour l'étude de la religion gauloise.

\section{SITUATION}

Le sanctuaire gaulois se trouve dans le village de Gournay-sur-Aronde, à l'intérieur d'un oppidum sur lequel nous reviendrons au paragraphe suivant. II n'est pas inutile de replacer la position géographique de cet ensemble. Gournay, accroché à la lèvre méridionale du plateau picard, est à l'intérieur de la cité des Bellovaques, dans une position quelque peu décentrée. Le trait le plus marquant du paysage paraît être la rivière d'Aronde, séparant puissamment le Bassin parisien du plateau picard. Ce rôle de frontière valut à l'habitat de Gournay une place privilégiée, si l'on peut dire, dans les grands conflits du Moyen Age. Mais il est probable que ce n'est pas cette seule caractéristique de la vallée qui poussa les Gaulois à élire ce site plus qu'un autre. L'intérêt économique de la rivière (1) est une raison déterminante. Le relief et la nature du terrain, enfin, autorisaient la construction d'une forteresse qui eût été difficilement réalisable ailleurs, dans la même région.

* Résidence de l'Abbaye, rue E. Branly, 60200 COMPIEGNE

** C.R.A.V.O, 21 rue des Cordeliers, 60200 COMPIĖGNE

\section{L'OPPIDUM (fig. 1)}

Ces raisons nous expliquent pourquoi l'oppidum fut construit là ; cependant, son origine demeure obscure, de même que son histoire. C'est afin d'éclairer l'une et l'autre que des fouilles ont été engagées en 1981, sur les fortifications précisément. L'oppidum a connu plusieurs phases d'édification, peut-être de multiples agrandissements. Tel qu'on peut le voir aujourd'hui, il se présente sous la forme d'un grand quadrilatère. Installé sur le versant sud de la vallée de l'Aronde, il s'appuie sur la rivière qui décrit en cet endroit une grande courbe. Deux longs fossés perpendiculaires séparent l'intérieur de l'oppidum du reste du plateau, sur les côtés sud et ouest. Vers le nord et l'est, la rivière et ses marais tourbeux eussent pu suffire à la défense, mais on leur avait adjoint une digue, retenant un étang de plus de six hectares. L'oppidum, pour sa part, et sous cette forme, possède une surface intérieure de 12 hectares. Mais il n'est pas impossible qu'il se soit poursuivi de l'autre côté de la rivière, occupant également le versant nord, là où se trouve le village actuel. Les fossés médiévaux entourant l'ancien bourg ont, en effet, pu reprendre des aménagements plus anciens (2).

Dans I'angle sud-ouest de ce grand quadrilatère se trouve une enceinte plus petite, dont le plan est également trapézoïdal. L'espace intérieur a une superficie de moins de trois hectares. C'est l'ensemble le plus puissamment défendu et dont la position est la plus dominante. S'il est possible que cette enceinte corresponde à l'état ancien de l'oppidum, il est certain que, par la suite, elle a gardé un rôle de redoute ultime. Une coupe stratigraphique (fig. 1, $n^{\circ} 5$ ) a permis 


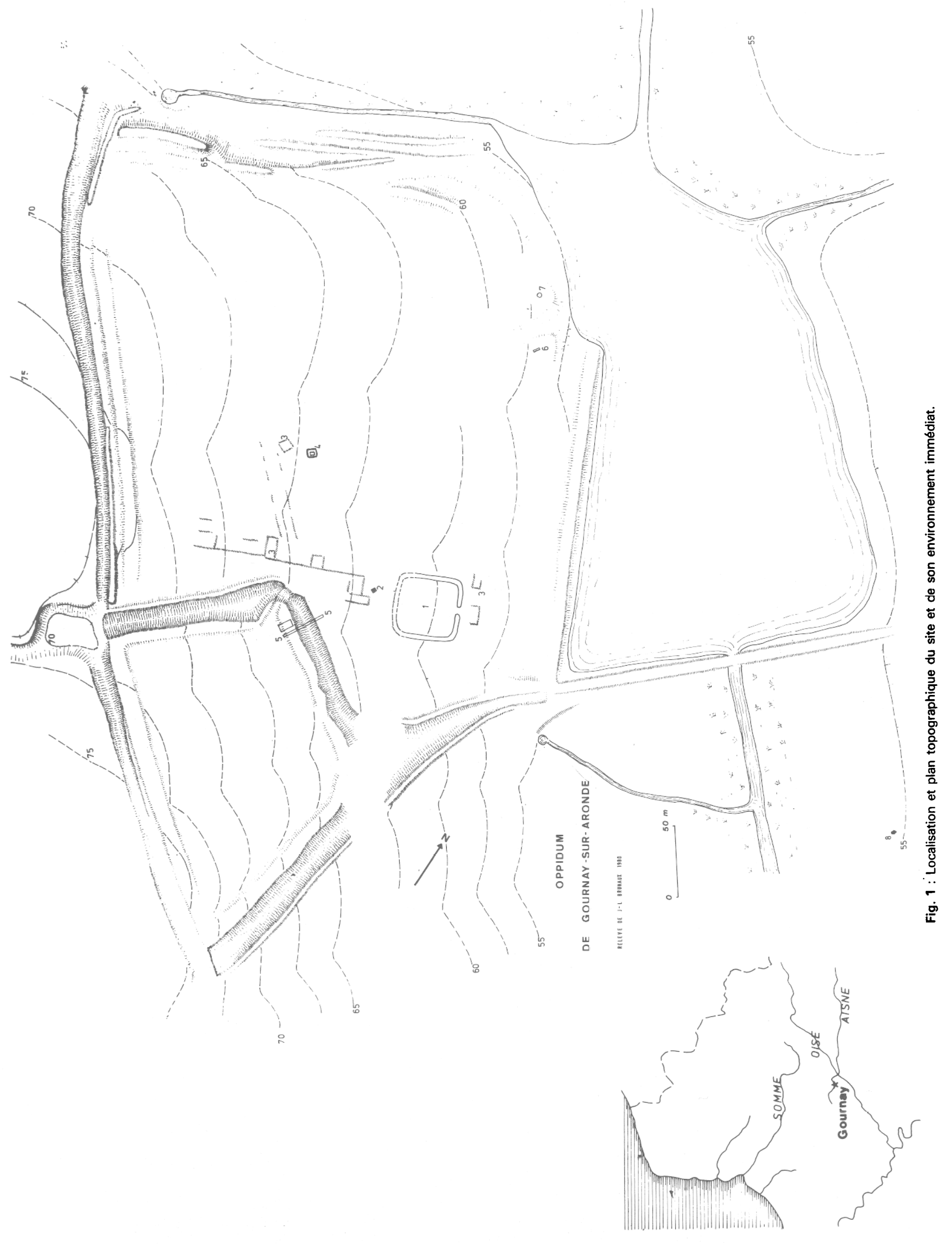


d'étudier la fortification sur le côté est, le seul où ce type de travail soit réalisable sans trop de peine, tant les fossés sont partout imposants. La coupe révèle qu'il y a eu, au moins, deux états du fossé. Le plus ancien datant peut-être du Chalcolithique, le plus récent ayant été comblé au début de la période galloromaine. De même, on observe deux états du rempart. La premier est constitué exclusivement de terre, il est large d'une dizaine de mètres, au minimum. Le second le recouvre, plus étroit (cinq mètres) ; il consiste en un noyau de terre retenue par deux murets de pierres sèches. A l'intérieur, des traces de poutres ont été observées. Deux grands clous de fer avaient été également découverts dans le fossé. Mais tant que ce matériel n'est pas retrouvé en place, il est prématuré de parler de murus gallicus. Les fortifications ont, en effet, été considérablement perturbées par une occupation gallo-romaine fort dense. Et ce n'est pas là un des aspects du site le plus négligeable. Car, contrairement à ce qu'on observe sur les grands oppida, chefs-lieux de cités, il semble qu'il n'y ait pas eu ici d'abandon de l'habitat, après l'invasion romaine.

\section{LE SANCTUAIRE}

Le sanctuaire se trouve à l'intérieur de l'oppidum, à cent mètres à l'est de la redoute que nous venons de décrire. Le relief du terrain semble également déterminant dans son emplacement : sur le flanc du versant méridional, il recouvre une coulée de limon en forme de butte, d'où l'on domine toute la vallée.

Le sanctuaire est, en fait, un enclos quadrangulaire de 45 mètres de côté. Un fossé matérialise ses limites. Cependant, dans la réalité, les choses sont plus complexes. Le fossé, étant très ancien, a précédé le sanctuaire proprement dit. Une interruption au milieu du côté est en marquait alors l'entrée. Dès le début du fonctionnement du sanctuaire en tant que tel, le fossé servit de réceptacle aux restes sacrificiels. II commença de se remplir; on dut remplacer cette matérialisation des limites de l'espace. Pour cela, une palissade fut installée sur le bord extérieur du fossé et, devant elle, on recreusa un nouveau fossé qui ne s'interrompait pas, face à l'entrée. II fallait utiliser une passerelle pour pénétrer dans le sanctuaire. Cet aménagement extérieur du sanctuaire persista jusqu'à la fin de l'Indépendance, mais connut quelques remaniements. La palissade dut être remplacée et le fossé extérieur fut, à plusieurs reprises, recreusé.

Cette limite artificielle (fossé ancien, puis palissade et fossé extérieur) avait pour fonction de séparer l'espace sacré, de forme carrée, du monde profane, en l'occurence l'habitat. Mais elle jouait également le rôle de contenant : à l'intérieur se trouvaient les installations proprement cultuelles sur lesquelles nous reviendrons.

\section{LE FOSSÉ A EXPOSITION}

Le premier fossé, le plus ancien - que nous appellerons "fossé à exposition" - a, dans le sanctuaire, une place exceptionnelle, certainement parce qu'il est la structure la plus riche d'informations : il a conservé une partie des restes sacrificiels, c'est-à-dire les seuls vestiges vraiment parlants et à partir desquels des rites se révèlent. Dans la réalité, d'autres structures, d'autres parties de l'ensemble devaieint avoir le même prestige.
Elles n'en ont gardé aucune trace, comme c'est souvent le cas pour les témoins archéologiques.

L'étude stratigraphique nous éclaire sur l'histoire du fossé. Un profil en $V$, profond, dans le meilleur des cas, d'1,80 m, large de $2,20 \mathrm{~m}$, c'est là sa forme ancienne, lorsqu'il n'avait pour fonction que d'être un fossé. Un temps assez long s'est écoulé avant qu'il ne devienne autre chose : il commença de se remblayer naturellement. Enfin, on l'aménagea solidement, très certainement lors de la "sanctuarisation" de l'enclos. Le fossé fut recreusé là où il était trop comblé et légèrement élargi. Mais surtout on le consolida chaque hiver (3), les parois s'éboulant lors du dégel. Pour remédier à cette fragilité, le fossé fut boisé sur toute sa longueur; des poutres furent placées longitudinalement dans le fond sur lesquelles on appuya des douves jointoyées. C'est de cette façon que le fossé put être utilisé pendant près de deux siècles.

Très vite, on commença de ranger dans cet immense réservoir les restes sacrificiels : armes, ossements. II s'agissait d'une véritable exposition dont la finalité ne peut être étudiée maintenant. Le rite s'est poursuivi pendant toute l'utilisation du sanctuaire : peu à peu le fossé s'est comblé. Mais cela ne s'est fait ni de façon uniforme, sur toute la longueur, ni régulièrement dans le temps. Un ordre, cependant, se dégage, qui sousentend des règles spatiales et temporelles concernant le choix des parties du fossé et la fréquence des dépôts. Ainsi, la zone entourant l'entrée se trouve entièrement comblée de deux gros amas, constitués chaque fois de six dépôts. La nature des restes déposés n'est pas non plus due au hasard: c'est là, par exemple, que se situent tous les crânes de bovidés. II existe donc une géographie sacrée de la structure. De la même manière, il y avait un calendrier des dépôts, dépendant, d'une manière plus générale, du férial, et soumis aux nécessités d'autres rites, sur lèsquels nous reviendrons.

Tel que nous l'avons observé en fouille, le fossé se présentait "grosso modo" de deux façons. II faut distinguer, en effet, les environs de l'entrée comblés par les deux amas déjà évoqués, du reste du fossé contenant des lits de matériel en alternance avec des lits de terre stérile. Le terme amas signifie ici l'empilement continu de six dépôts spécifiques qui remplissaient le fossé, du fond à la surface labourée. Là, les objets étaient littéralement imbriaués les uns dans les autres. Et les six ensembles ne se distinguaient ni en fouille ni en stratigraphie (4). Les grands objets avaient souvent un fort pendage, manifestant par leur position le soin qu'on avait pris à les exposer: plaqués contre les parois et serrés les uns près des autres.

Sur le reste du fossé, le matériel figurait en nombre moins important, la densité demeurait cependant impressionnante. Suivant les secteurs, celle-ci variait, ainsi que la nature des objets, confirmant par là l'existence d'une géographie préexistant aux dépôts.

Au total, 2800 ossements et 2200 objets ou fragments métalliques furent découverts. Pour ces derniers, le décompte provisoire donne : 100 épées, 150 fourreaux d'épées, 220 umbos de boucliers, 70 fers de lance, 40 talons de lance, 80 fibules, 70 chaînes de ceintures et de nombreux éléments séparés : anneaux, agrafes, clous, tiges, rivets et fragments divers. 
Le matériel, pour la plus grande part, a été sacrifié. En ce qui concerne les ossements, nous le verrons plus bas. Les armes, elles, le montrent à l'évidence : les épées sont pliées, cassées, coupées. Leurs fourreaux sont démontés, tordus, morcelés. Les umbos de boucliers sont enfoncés, martelés, percés, découpés. II n'est pas de petits objets qui n'aient subi le même sort : les fibules sont ouvertes, repliées. Cette violence sacrificielle est polymorphe : elle tient à la nature des objets sacrifiés et à celle des instruments utilisés dans ce dessein : marteau, hache, épée, lance, tenailles, etc. En ce sens, le matériel archéologique de Gournay est exceptionnel, à double titre parce qu'il présente une production comparable au site éponyme de La Tène et parce qu'en plus il donne à voir le plus impressionnant catalogue d'armes sacrifiées.

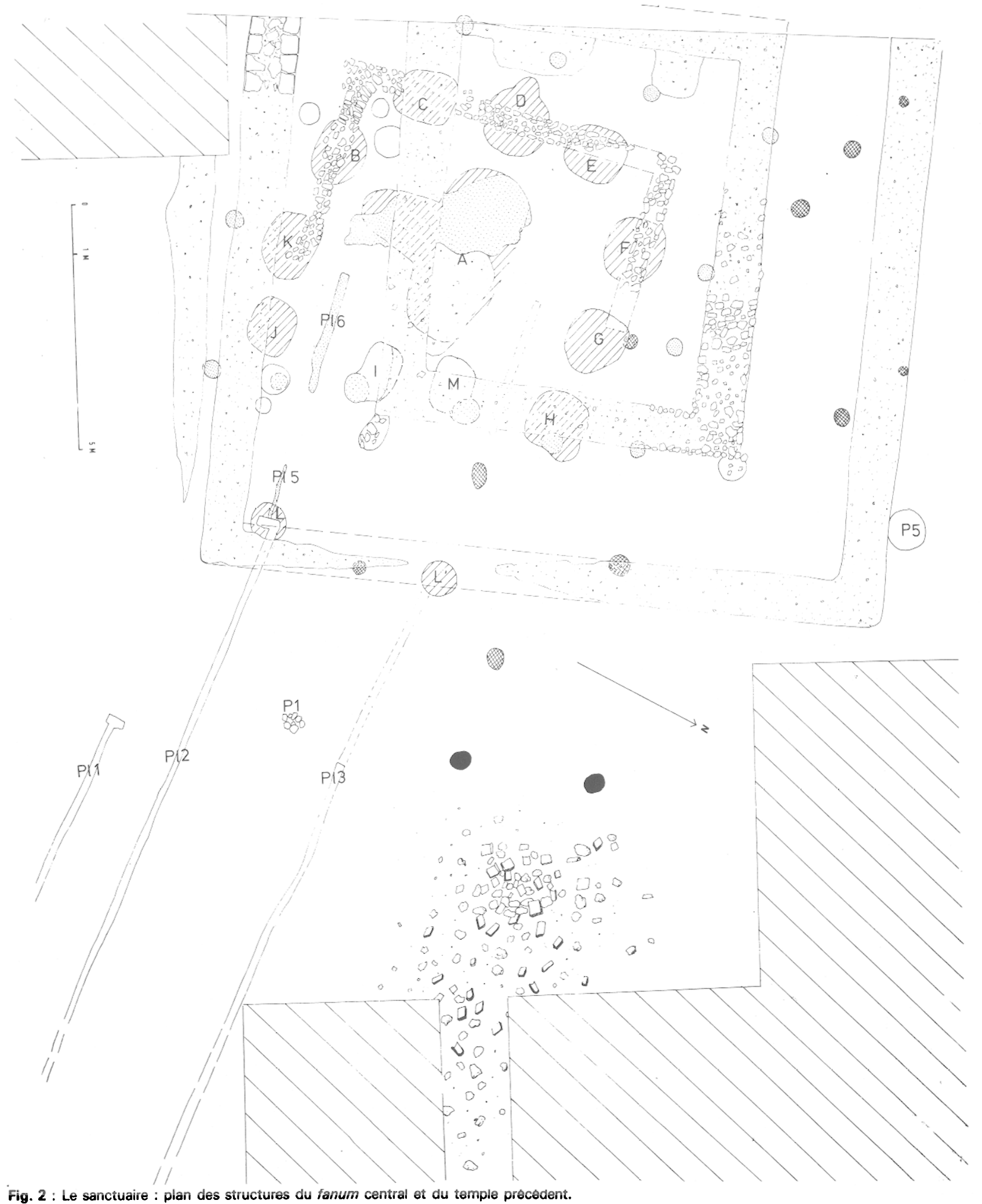


Cette mise en scène des restes sacrificiels que nous venons de décrire rapidement a une fonction. Elle est la suite, l'aboutissement même d'une longue série de rites. Pour les retrouver, et comprendre cette fin des restes sacrificiels, il faut maintenant se tourner vers l'intérieur du sanctuaire.

\section{LES STRUCTURES INTERNES}

C'est, en effet, en fouillant l'espace intérieur de l'enclos que bien des étrangetés, si elles n'ont pu être totalement expliquées, ont néanmoins été restituées à l'intérieur d'un phénomène plus large, celui de la liturgie sacrificielle. Mais examinons auparavant comment se présente archéologiquement cet espace. On peut le diviser en trois parties : une zone centrale où se trouvent les structures cultuelles, un tiers sud vierge de tout vestige gaulois, un tiers nord riche en trous de poteaux et piquets.

La zone centrale nous retiendra plus particulièrement. Au centre géométrique de l'enclos sont accumulés les vestiges de cinq ensembles successifs. On se doute qu'il n'est pas possible en quelques lignes de préciser la méthode utilisée pour les différencier, comme il est impossible de décrire dans le détail chacun des ensembles et de le mettre en rapport avec l'état du sanctuaire contemporain. L'ensemble le plus ancien est un groupe de dix fosses : neuf sont disposées en cercle autour d'une dixième plus importante. Elles avaient une fonction cultuelle que nous évoquerons au paragraphe suivant. Seule la fosse centrale survécut à ce premier ensemble (5) ; dans chacune des fosses périphériques on installa un trou de poteau pour édifier un temple sub-ovalaire, possédant une façade à l'est (cf. fig. 3). II abritait la grande fosse qui resta utilisée jusqu'à la Conquête. Le temple qui lui succéda adopta la forme carrée (cf. fig. 4). II gardait la façade à l'est; nous savons que les trois autres côtés étaient fermés par des cloisons soutenues par des piquets. Ce temple fut détruit, ainsi que d'autres structures, par un incendie (6).

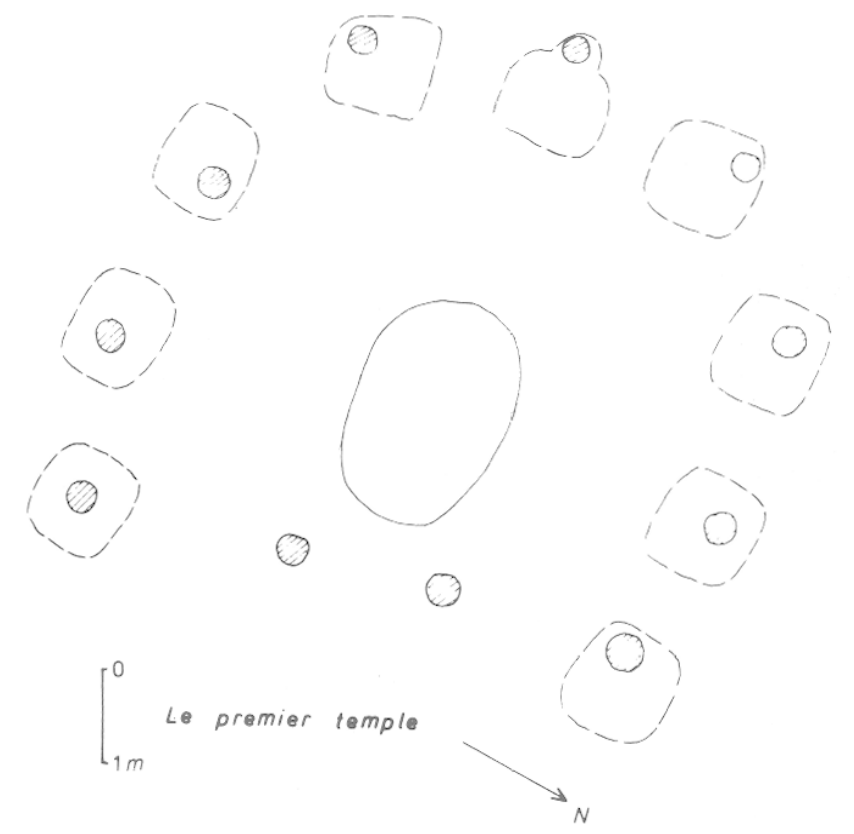

Fig. 3 et 4 : Etats successifs du temple qui a précédé le fanum.
Le troisième temple diffère sensiblement des précédents (cf. fig. 2) : il possède une cella en pierres sèches, entourée d'une galerie légère en bois, se terminant sur une façade ouverte à l'est. La fosse centrale est alors utilisée comme grand foyer. Si les deux premiers temples précèdent la Conquête, ce dernier lui est postérieur, bien que l'influence romaine n'apparaisse que faiblement dans l'architecture.

Ce temple, véritable prototype du fanum, ne connut certainement pas une longue utilisation. II dut succomber aux mesures de Claude interdisant les rites druidiques. Pendant près de trois siècles, le sanctuaire resta désert, alors que de toutes parts il était environné de structures gallo-romaines. Cependant; et aussi étonnant que cela puisse paraître, à la fin du IIle siècle un véritable fanum fut édifié à l'emplacement exact des temples gaulois (cf. fig. 2). Cela suppose une mémoire collective du lieu de culte ou la pratique persistante de rites interdits. A nouveau, le temple fut totalement arasé. Cette fois, aucune structure cultuelle ne vint lui succéder.

Nous venons de voir les structures principales à l'intérieur du sanctuaire. Celles-ci s'intégraient à un ensemble organisé dans lequel intervenaient les vestiges du tiers nord. Ces derniers consistent en trous de poteaux, piquets, traces fossiles de branchages et fosses, accumulés sur deux siècles. Pour les étudier, il faudrait entreprendre une description de chacun des états du sanctuaire; nous ne pouvons que renvoyer le lecteur à la publication définitive.

\section{LES RITES}

La répartition du matériel dans le fossé, l'étude des structures internes et l'analyse du matériel sacrifié permettent, non pas de reconstituer des rites, mais de mettre en évidence des fragments de ces derniers : gestes, séries d'actions rituelles qui resteront évidemment pour nous énigmatiques.

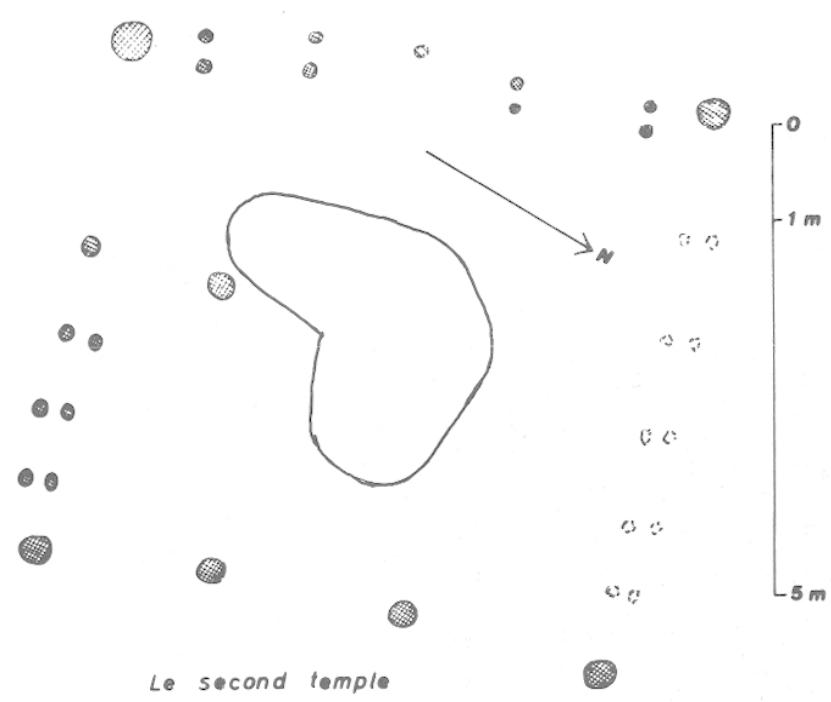


Le rite principal, celui autour duquel tout s'ordonne, est bien entendu le sacrifice. A Gournay, sa présence est confirmée par des vestiges multiples : la plupart des objets métalliques, les ossements dans leur ensemble et plus particulièrement ceux qui portent la trace de coups violents ayant entraîné la mort. Habituellement, on distingue les sacrifices suivant la divinité à laquelle ils s'adressent, ou suivant le bénéfice qu'ils sont censés apporter. De telles distinctions ne sont pas possibles ici. Nous ne savons rien des divinités adorées dans le sanctuaire; la découverte archéologique, d'une manière générale, est incapable de révéler la finalité subjective d'actes rituels. Seule la nature des victimes permet un premier classement des sacrifices. Au nombre de trois, c'est dans un ordre hiérarchique : I'homme, l'animal et l'objet. Du sacrifice humain nous avons, cette fois, la preuve incontestable sous forme de vertèbres sectionnées. Onze victimes, au moins, auraient péri de la sorte. Le sacrifice animal est plus complexe; il comprend les bovidés, les équidés, les ovins, les porcins et les chiens. La mise à mort peut varier pour une même espèce; des coups de nature différente ont parfois été assénés sur le même crâne. II n'est pas possible d'entreprendre mairitenant la description détaillée des différents types. de mise à mort. Pour les armes, nous avons vu plus haut et brièvement ce qu'il en était.

Une autre série d'informations tient à la destinée du matériel sacrifié, zone habituellement très floue de la connaissance des religions. Les rapporteurs - c'est le cas de César - se sont contentés de décrire le sacrifice proprement dit ou ce qui le précédait. Or, généralement - nous le savons pour Rome et la Grèce où des rites séparés dans le temps réutilisaient les mêmes restes sacrificiels (Fordicidia, Thesmophories) - la victime, une fois sacrifiée, doit encore suivre un long périple. L'analyse des restes osseux et la fouille de l'initérieur du sanctuaire permettent de restituer quelques phases de ce parcours.

Tous les objets du sacrifice n'ont pas forcément suivi le même chemin. Des sacrifices entiers ont pu ne laisser aucune trace archëologique. D'autres n'ont abandonné aux fouilleurs qu'une partie de la victime (7). Le développement que nous donnons ci-après n'est donc que formel.

- La victime mise à mort lou détruite pour les objets) est déposée dans l'une ou plusieurs des fosses centrales, auprès desquelles le sacrifice devait avoir lieu.

- On la laissait, pour qu'elle pourrisse ou qu'elle rouille pendant plusieurs mois, le temps pour les animaux que la décomposition soit suffisamment avancée (que seule la colonne vertébrale soit encore solidaire). fosse.

- La victime était soigneusement exhumée de la

Alors, deux possibilités, au moins, se présentaient :

- ou on la déposait directement dans le fossé à exposition.

- ou on l'exposait à l'intérieur du sanctuaire, pendant un temps fort long, à la suite duquel on la déposait dans le mêrne fossé.

- une troisième possibilité semble devoir être retenue : celle d'un sacrifice recommencé sur l'un des restes de la victime (crâne notamment).
A la suite de ces manipulations, le "reste" sacrificiel prenait une place définitive, éternelle, pour ainsi dire, dans le fossé. Cette place, comme nous l'avons vu plus haut, ne devait rien au hasard. Des règles définissaient une partition de l'espace. Quant au fossé luimême, comblé de ces richesses, il participait à la clôture du sanctuaire, de manière symbolique, les restes sacrificiels étant certainement chargés d'une vertu apotropaïque.

\section{LE SANCTUAIRE DE GOURNAY ET LA RELIGION GAULOISE}

Après cette description rapide, il serait prématuré de conclure. II nous semble important de définir l'utilisation qu'on peut faire de cette somme d'information, là où elle semble pertinente: dans le domaine de la religion gauloise.

En ce sens, les travaux sur Gournay apportent une documentation plus anthropologique qu'historique. On ne doit pas s'attendre à découvrir un type de sacrifice bien précis (même si des similitudes avec le suovetaurilia apparaissent), encore moins des données sur le panthéon. Nous ignorons l'identité du dieu auquel les victimes étaient dédiées, ainsi que le cadre dans lequel elles l'étaient. De la découverte seuls surgissent quelques fragments d'une liturgie qu'on doit croire aussi complexe que celle d'autres religions antiques. On sait qui était sacrifié dans le sanctuaire du Parc, comment il l'était, ce que devenait la victime après le sacrifice. Par ailleurs, se révèle, après la découverte de Libenice, le second exemple de ce qu'est un sanctuaire gaulois, appartenant, cette fois à part entière, à l'époque de La Tène.

Par delà la découverte archéologique exceptionnelle confiée maintenant aux typologues - c'est une nouvelle approche de la société gauloise qu'il faut imaginer. De celle-ci, l'analyse des vestiges du Parc nous donne des images pleines de sens. A travers le matériel sacrifié, ce sont bien les trois grandes classes de la société qui se révèlent: les equites, sous forme des armes, symbole à la fois de leur puissance militaire et de leur contribution effective au fonctionnement de la société (défense et agrandissement du territoire); les paysans, dont une partie de la production - bétail, mais aussi végétaux qui n'ont laissé aucune trace est consommée religieusement, tandis que l'autre partie l'est socialement; druides enfin, qui assurent le brassage de ces biens, qui en accomplissent la fusion, celle-ci réalisant symboliquement, mais aussi réellement, au moment du sacrifice, la fusion des classes, à travers le prisme d'une richesse s'accumulant.

\section{LES ANIMAUX DU SACRIFICE}

Le fossé entourant le sanctuaire de Gournay-surAronde a livré des vestiges de sacrifice, parmi lesquels figurent un ensemble d'environ trois mille ossements animaux et humains. Les ossements humains sont étudiés par F. POPLIN, du Muséum National d'Histoire Naturelle (Paris). Les ossements animaux décrits ici proviennent d'espèces domestiques courantes: le bœuf (Bos taurus), le cheval (Equus caballus), le mouton (Ovis aries) et le porc (Sus domesticus). Le chien est présent dans un remplissage particulier que nous n'étudierons pas dans ce résumé. Trois espèces sauvages, le lièvre (Lepus europaeus), le canard colvert 
(Anas platyrhynchos) et la corneille noire (Corvus corone), sont représentées chacune par un os.

II n'est évidemment pas possible, en quelques pages, de rassembler la totalité de l'information livrée par les ossements. Cela fait l'objet de la publication générale (MENIEL P., à paraître). Dans ce qui suit, nous donnerons les principales caractéristiques des animaux sacrifiés, telles que la taille, l'âge, le sexe, ... et que les ossements nous permettent de déterminer ou d'estimer. Ces particularités sont inhérentes à une structure anatomique que le sacrifice est venu modifier. Ces modifications constituent un ensemble de données archéologiques que nous décrirons ensuite.

\section{Les caractéristiques des animaux sacrifiés}

L'approche zoologique est principalement basée sur l'étude biométrique des os longs et des crânes. Elle permet d'établir l'importance du dimorphisme sexuel, de mettre en évidence l'influence du milieu environnant sur l'élevage et de suivre son évolution au cours des âges. Une autre partie est consacrée à l'estimation des âges et des sexes, à l'aide de la méthode précédente, ou d'examens visuels divers.

\section{1. Age des animaux sacrifiés}

Les âges d'apparition ou de remplacement des différents types de dents sont connus, tout au moins pour les principales espèces domestiques. Malgré tous les écarts qui sont susceptibles de perturber l'estimation, cette dernière reste la meilleure que l'on puisse réaliser. D'autres méthodes sont utilisables. Celle qui est basée sur l'examen de l'usure permet de calculer l'âge des animaux adultes, elle prend ainsi le relai de la précédente. Elle nécessite des données de références; nous utilisons celles de LESBRE (1920) pour le cheval, celles de DUCOS (1968) pour les ruminants et celles de VARIN (1980) pour le porc.

La mise en œuvre de ces estimations nous permet de tracer les diagrammes de la figure 5 .

II apparaît sur cette figure que les bœufs sont très âgés. L'âge moyen des animaux sacrifiés est d'environ 9 ans. Cette moyenne élevée est due à la présence d'animaux dont les dents sont réduites à des chicots, et qui sont donc âgés de treize ans au moins.

Pour les chevaux, l'âge moyen est d'environ dix ans, mais là encore on note la présence d'individus d'âge élevé.

Ces deux espèces n'ont pas été consommées, alors que le porc et le mouton l'ont été, comme le prouvent les nombreuses traces de découpe sur la surface des os. Nous ne connaissons évidemment pas les bénéficiaires de cette consommation. Quoi qu'il en soit, pour les porcs, ce sont les animaux jeunes, voire très jeunes, qui prédominent. II n'est pas possible de parler d'un âge d'abattage préférentiel, comme c'est souvent le cas sur les sites d'habitat de la région. Les moutons n'ont presque pas livré de mâchoires, il faut donc recourir à une autre méthode de détermination des âges, basée sur l'état de développement des os longs.

En effet, le développement d'un os long se fait en plusieurs phases, une de celles-ci correspond à la soudure des extrémités articulaires (épiphyses) sur le corps de l'os (diaphyse). Ces dates de sutures sont connues (BARONE, s.d. et SCHMID, 1972), on peut
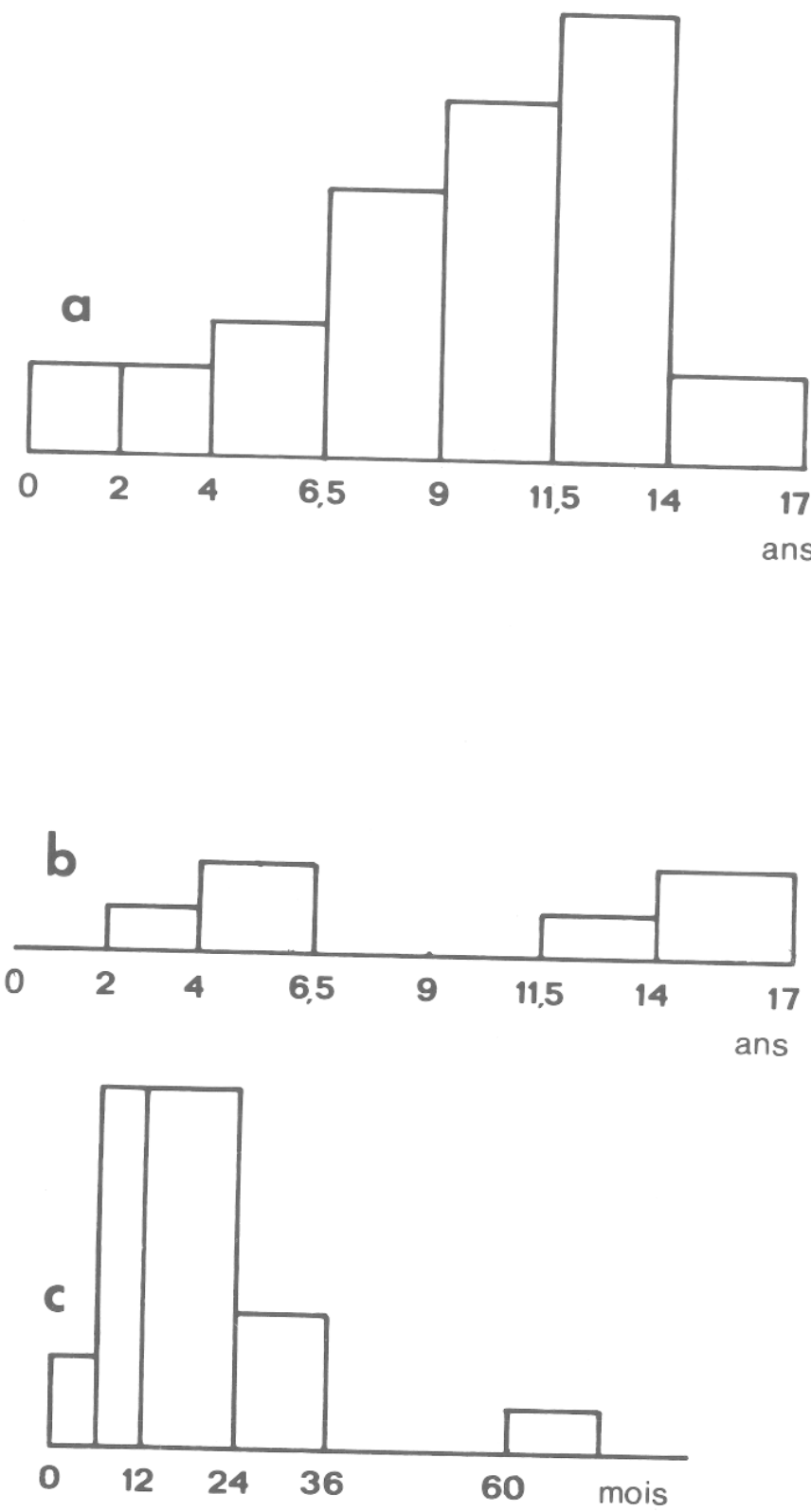

Fig. 5 : Détermination des âges de mise à mort par l'exameń des dentitions - $a$, âge de mise à mort des bœufs ; - b, des chevaux : des porcs.

donc attribuer chaque os à l'une des trois classes d'âges que les dates de soudure des deux extrémités nous permettent de définir. Dans certains cas particuliers cette méthode permet de déterminer l'âge effectif d'un animal, lorsqu'une soudure est en cours, par exemple. Appliquée aux restes de mouton et de bœuf, ces estimations nous donnent les résultats de la figure 6.

II apparaît que les moutons sont très jeunes : plus de la moitié des soixante dix individus a moins de quatre mois.

Pour le bœuf, les résultats ont été simplifiés, en ne considérant qu'une limite, celle de quatre ans, et en répartissant les résultats donnés par chaque type $d^{\prime}$ os de part et d'autre de cet âge. Nous y avons reporté aussi les résultats obtenus lors de l'examen des dents sur une échelle équivalente. Une organisation très prononcée des os absents est révélée par cette repré- 
sentation, les animaux jeunes sont représentés de façon équivalente par leurs mâchoires et par leurs os longs, mais il manque leurs crânes, alors que pour les adultes il manque de nombreux os de membres ...
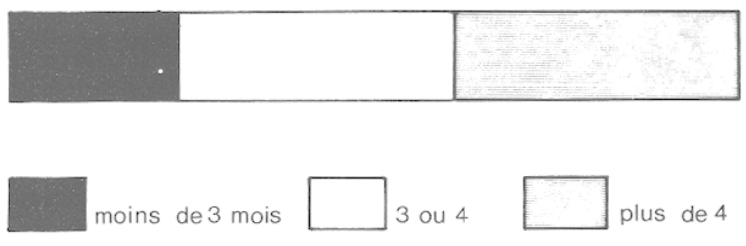

a

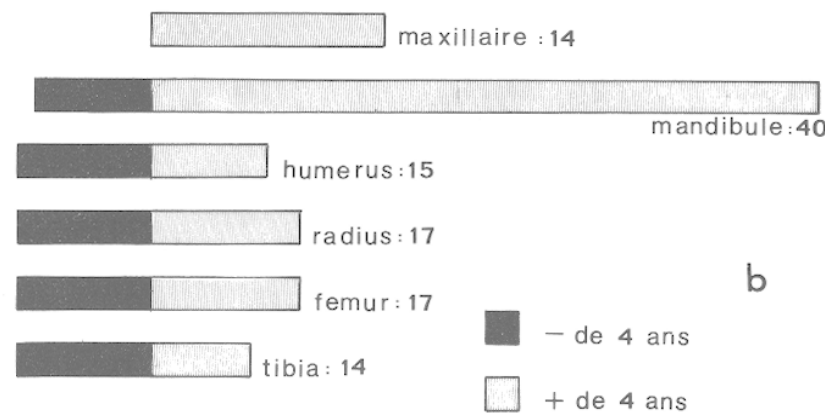

Fig. 6 : Détermination de l'âge de mise à mort des moutons à partir du degré de développement des mâchoires et des os longs des membres.

\section{2. Etude des sexes}

La détermination de la jument et de l'étalon ne pose pas trop de problème lorsque des mâchoires sont conservées. La présence de canines caractérise les étalons, au nombre de quatre pour deux juments dans le remplissage du fossé. Pour les deux autres cette détermination, faute de mâchoires, n'a pas été possible.

Pour les porcs, c'est encore la canine qui sert, non plus par sa présence éventuelle, mais par sa morphologie et son mode de croissance. Encore faut-il que cette dent soit définitive, c'est-à-dire que le porc dont on veut déterminer le sexe ait dépassé un an. Seuls une dizaine d'animaux. remplissent cette condition, ils se répartissent en six femelles et quatre mâles.

Pour les ruminants, la détermination du sexe n'est pas aussi simple. Les chevilles osseuses permettent une détermination pour des individus caractéristiques, mais il subsiste souvent un bon nombre de cas douteux. Les métapodes, par leur gracilité, sont caractéristiques des sexes, mais ils sont peu nombreux à Gournay. Par contre, les bassins sont plus nombreux, mais on se heurte là aussi à de nombreux cas litigieux, qui ne permettent pas de compléter les déterminations précédentes. La proportion de vaches, déterminée avec ces diverses méthodes, est comprise entre un quart et un tiers du nombre total d'individus, soit dix à treize vaches pour une trentaine de males (bœufs et taureaux).

\section{3. Taille des animaux}

La taille des animaux est exprimée par la hauteur au garrot. Cette dimension peut être estimée à partir de la longueur de certains os, différents selon les espèces, et de facteurs multiplicatifs particuliers. Nous avons utilisé ceux publiés par BOESSNECK (1971).
On obtient ainsi les résultats ci-dessous :

\begin{tabular}{|lccc|}
\hline & sexe & nombre & hauteur $\mathrm{cm}$ \\
\hline bœuf & & 2 & 117 \\
& & 11 & 120 \\
cheval & & 2 & 127 \\
& & 3 & 131 \\
mouton & & 6 & 61 \\
porc & 5 & 5 & 65 \\
\hline
\end{tabular}

Ces données s'inscrivent dans celles obtenues par ailleurs sur les sites d'habitat de la région, ce qui semble exclure une sélection d'animaux particulièrement grands, comme ce sera le cas, quatre siècles plus tard, au même endroit (8).

\section{Caractéristiques archéologiques}

L'importance numérique et relative des différentes espèces peut être illustrée par les données ci-dessous :

\begin{tabular}{|lcc|}
\hline & $\begin{array}{c}\text { Nombre } \\
\text { de restes }\end{array}$ & $\begin{array}{c}\text { Nombres minimaux } \\
\text { d'individus }\end{array}$ \\
\hline bœuf & 1300 & 42 \\
cheval & 370 & 8 \\
mouton & 600 & 100 \\
porc & 180 & 40 \\
\hline
\end{tabular}

Les ossements sont en grande majorité très bien conservés, peu ou pas fragmentés, mais présentent cependant des différences d'aspect selon les endroits du fossé dont ils proviennent. La plupart des restes de bœuf provient de deux riches amas situés de part et d'autre de l'entrée du sanctuaire, et si certains sont très frais, en couleur et en état de surface, d'autres, par contre, présentent les traces d'une attaque corrosive qui en modifie la coloration. Cette attaque est localisée sur une face ou sur une extrémité des os qu'elle a touchés, de plus on ne l'a observée qu'en certains endroits des dépôts. Ces derniers sont constitués, entre autres, par de nombreux fragments de rachis en connexion; ces ensembles, une fois déposés, n'ont plus été déplacés, car cela les aurait irrémédiablement disloqués. D'autre part, l'attaque est organisée selon des plans, correspondant à un découpage en dépôts élémentaires des deux amas. Elle est donc intervenue sur les dépôts en place dans le fossé. On arrive ainsi à établir, après coup, une partition verticale des-amas, alors que la fouille ne les avait pas mis en évidence. De plus, cette attaque est dûe à I'action des agents atmosphériques qui, compte tenu de l'état des os, sont restés plusieurs années en contact avec les dépôts, sans que ceux-ci soient recouverts par d'autres matériaux.

Mais ce qui est valable pour les restes de bovidés, ne l'est pas pour les équidés. Les chevaux sont isolés les uns des autres, non pas par squelettes entiers (considérés du point de vue des relations anatomiques ou de la composition en ossements), mais par ensemble de restes d'un même animal, contenant de vingt à cent pièces selon les cas. II subsistait, à l'intérieur de ces ensembles, quelques connexions anatomiques au sens strict, et des relations de voisinage proches de 
l'articulation bougée. Cette conservation partielle des structures anatomiques est troublante, car les os trouvés isolés ne portent aucune trace de découpe (les seules que nous ayons observées ne visaient pas les articulations). D'autre part, ces relations partielles trouvent des similitudes chez le bœuf, de qui une centaine d'ensembles de vertèbres en connexions a été dégagée.

L'hypothèse d'une décomposition naturelle, a priori plus satisfaisante que la cuisson dans l'eau bouillante, a été confirmée par une étude expérimentale réalisée sur des cerfs. II nous a ainsi été possible de montrer que les relations anatomiques relevées sur les os de bœufs et de chevaux correspondent à celles observées pour une des phases finales de la dislocation d'un cadavre décomposé. Cette observation implique un réseau de déductions qui nous permettra une reconstitution partielle du sacrifice animal.

Ces caractéristiques originales des vestiges animaux du sanctuaire de Gournay, associées à des indices concrets, tels que des traces de coups de hache sur la face nuquale des crânes de bœufs, font de cet ensemble un objet de choix pour l'étude archéozoologique. Cette dernière permettra de reconstituer, à partir d'animaux dont elle décrit partiellement l'aspect et les caractéristiques, plusieurs étapes du sacrifice, et parmi elles la dernière, qui est à l'origine des dépôts découverts et fouillés. Tandis que d'autres étapes resteront irrémédiablement dans l'ombre.

\section{NOTES}

(1) cf. communication au $6^{\text {e }}$ colloque sur le deuxième Age du Fer en France non méditerrannéenne Mons-Bavai 1982. BRUNAUX J.-L. "L'oppidum du Parc à Gournay-sur-Aronde".
(2) Cela cependant reste difficile à démontrer. Les remaniements dans ce secteur, ont été nombreux, du début du Moyen Age jusqu'à nos jours.

(3) Nous avons pu observer le phénomène, alors que le fossé fouillé demeurait ouvert.

(4) Ils ont dû être déterminés par d'autres méthodes : observations ostéologiques essentiellement.

(5) II s'agit du premier ensemble repérable. La fosse centrale a pu, pendant un temps, rester seule, les fosses périphériques s'ordonnant, par la suite, autour d'elle.

(6) En ce qui concerne I'historique du sanctuaire, nous renvoyons à la publication définitive.

(7) Ainsi que le prouve le décompte des ossements.

(8) Une favissa a été découverte à 150 mètres au nord-est du sanctuaire. Elle est datée du deuxième siècle après J.-C. et contenait de nombreuses cornes de bovidés d'une taille exceptionnelle, visiblement sélectionnés pour leur beauté.

\section{BIBLIOGRAPHIE}

BARONE, R., s.d. Anatomie comparée des mammifères domestiques, Vigot, Paris.

BOESSNECK J., (1971) Die Tierknochenfunde aus dem Oppidum von Manching, Band VI, Franz Steiner Verlag, Wiesbaden.

BRUNAUX, J.-L. (1983) Le sanctuaire celtique de Gournay-surAronde et la religion gauloise. Thèse de Ille Cycle, Université de Tours 1982 (publication prévue pour 1983).

DUCOS, P. (1968) L'origine des animaux domestiques en Palestine, Imprimeries Delmas, Bordeaux.

LESBRE, F.-X. (1920) Précis d'extérieur du cheval et des principaux mammifères domestiques, Asselin et Houzeau, Paris.

MENIEL, P. (à paraître) Les animaux du sacrifice dans le sanctuaire gaulois de Gournay-sur-Aronde.

SCHMID, E. (1972) Atlas of animal bones, Elsevier publishing company, Amsterdam, London, New York.

VARIN, E. (1980) Chevreuil, cerf et sanglier, Les Editions de l'Orée, Bordeaux. 